Reprod. Nutr. Dévelop., 1988, 28 (6 A), 1519-1530

\title{
Effets d'une carence en acides gras essentiels sur la composition en acides gras des muscle, foie et tissu adipeux chez la truite femelle (Salmo gairdneri) au cours du cycle sexuel
}

\author{
I. NASSOUR, C. L. LÉGER
}

Station de Recherches de Nutrition, I.N.R.A., 78350 Jouy-en-Josas, France.

Summary. Effects of an essential fatty acid deficiency on the fatty acid composition of muscle, liver and adipose tissue in female trout (Salmo gairdneri) during sexual cycle.

Fatty acid composition of adipose tissue, muscle and liver were analyzed in trouts fed either a n-3 enriched control diet or a n-3 deficient diet during the last two months of ovarian development. Feeding the $n-3$ deficient diet did neither modify the hepatic level of total lipids (LT) nor the proportion of neutral lipids (LN) and polar lipids (LP). However, this diet produced a significant decrease in muscular $L T$ due to a $L N$ decrease. Levels of saturated fatty acids in liver, muscle and adipose tissue were not markedly modified, in contrast to those $n-7$ and $n-9$ which were highly dependent on the nature of diet, in particular in LN fraction. In muscle and liver $n-3$ fatty acid levels were found to decrease in $L N$ and LP fractions when animals were fed the deficient diet. The $n-3$ decrease was found to be compensated by $n-6$ fatty acid level in LP fraction and by $n-9$ and $n-6$ levels in $L N$ fraction. In adipose tissue, the levels of major $n-3$ fatty acids $(20: 5 n-3$ and $22: 6$ $n-3)$ and total $n-3$ fatty acids were not significantly modified by the $n-3$ deficient diet during the experimental period. These results might be associated to a modification in the function of adipose tissue during this period.

\section{Introduction.}

Le besoin en acides gras essentiels (AGE) des poissons varie considérablement d'une espèce à l'autre (Castell, 1979 ; Léger, 1980). De nombreuses études ont montré que les truites Salmo gairdneri ont besoin dans leur alimentation d'acide linolénique (18:3n-3) ou des acides gras longs polyinsaturés en $n-3$ $(C \geqslant 20$, nommés AGLPI $n-3)$ pour réaliser une croissance normale et faire disparaître les symptômes de carence (Yu et Sinnhuber, 1972, 1975 ; Castell et al., 1972 a, b, c ; Watanabe et al., 1974 a, b, c ; Takeuchi et Watanabe, 1976, 1977 a). Cependant, la carpe Cyprinus carpio (Watanabe et al., 1975) et I'anguille Anguilla japonica (Takeuchi et al., 1980) semblent avoir également besoin de l'acide linoléique (18:2 n-6). Chez la truite, les AGLPI n-3 ont une "activité » AGE deux fois plus élevée que celle du 18:3n-3 (Watanabe et Takeuchi, 1976 ; 
Takeuchi et Watanabe, 1977 b). La différence d'activité AGE entre les acides gras polyinsaturés (AGPI) à $18 \mathrm{C}, 20 \mathrm{C}$ ou $22 \mathrm{C}$ provient du fait que l'animal convertit les acides à $18 \mathrm{C}$ en acides à 20 et $22 \mathrm{C}$ portant un plus grand nombre de doubles liaisons, grâce à un système de bioconversion comportant des enzymes limitantes, les désaturases (Owen et al., 1975; Léger et al., 1979). Les poissons qui possèdent un équipement défectueux en $\Delta 6-, \Delta 5$ - et $\Delta 4$-désaturase doivent trouver dans leur alimentation les AGLPI $n-3$, car ils sont incapables de les synthétiser en quantités suffisantes à partir du $18: 3 n-3$. Ce n'est pas le cas de la truite : quand ce poisson est soumis à un régime contenant le $18: 3 \mathrm{n}-3$ comme seule source lipidique, les processus de reproduction et de développement embryonnaires et larvaires sont assurés normalement (Yu et al., 1979).

$L$ 'influence de la carence en AGE sur le contenu et la composition en acides gras (AG) des lipides tissulaires a été largement étudiée chez la truite au stade alevin (Castell et al., 1972 a ; Yu et Sinnhuber, 1972, 1975 ; Watanabe et al., 1974 a, b, c ; Takeuchi et Watanabe, 1976, 1977 a, b). Par contre, peu de travaux ont été consacrés à ce thème chez la truite femelle en période de la reproduction (Léger et al., 1981 ; Watanabe et al., 1984 ; Leray et al., 1985).

Nous avons étudié dans le présent article l'influence d'une carence en AGE pendant les deux mois précédant l'ovulation sur la teneur et la composition en acides gras du foie, du muscle et du tissu adipeux. Une telle étude est importante lorsque l'on sait que la mobilisation des réserves lipidiques de l'adulte contribue pour une part importante aux réserves de l'œuf (Nassour et Léger, données non publiées) et que de la qualité de ces dernières dépend le bon déroulement de l'éclosion et du développement embryonnaire et larvaire (Leray et al., 1985).

\section{Matériels et méthodes.}

Animaux. - Le travail est réalisé sur des truites arc-en-ciel Salmo gairdneri, âgées de 2 ans et élevées en pisciculture expérimentale à une température de 10 à $12^{\circ} \mathrm{C}$. Ces animaux sont nourris dès la résorption du sac vitellin et jusqu'à la période expérimentale avec un régime commercial $(C)$ contenant $8 \%$ de lipides riches en AG $n-3$. Au début de l'expérience (11 septembre), 6 femelles sont sacrifiées (poids : $765 \pm 50 \mathrm{~g}$ ), puis le tissu adipeux périviscéral (TAP) et un échantillon de muscle sont prélevés. Les animaux sont divisés ensuite en deux groupes de 20 poissons d'un poids moyen de $800 \mathrm{~g}$. Le premier groupe (M) est nourri avec un aliment expérimental contenant $10 \%$ d'huile de foie de morue riche en AG n-3 ; le second groupe (S) a reçu un aliment semblable où l'huile de foie de morue était remplacée par du saindoux déficient en AG $\mathrm{n}$-3. Les constituants et la composition en acides gras de l'aliment sont présentés dans les tableaux 1 et 2 . Dès le 11 septembre (ovocytes au cinquième de leur développement final en volume) et jusqu'à l'ovulation, le 17 novembre, les animaux sont pesés tous les 15 jours, afin d'ajuster le poids d'aliment distribué journellement au taux de $1 \%$ du poids vif. Après la ponte, dans chaque groupe, 6 femelles sont sacrifiées (poids: $1024 \pm 88 \mathrm{~g}$ pour le lot $\mathrm{M}, 1010 \pm 77 \mathrm{~g}$ pour le lot $\mathrm{S}$ ) puis le muscle, le foie et le TAP sont prélevés individuellement pour analyse. 
TABLEAU 1

Composition en acides gras de l'aliment.

\begin{tabular}{|c|c|c|c|}
\hline Aliment & C & $M$ & $S$ \\
\hline $14: 0$ & 3,3 & 3,2 & 1,3 \\
\hline $16: 0$ & 20,5 & 11,2 & 26,2 \\
\hline $18: 0$ & 9,2 & 2,6 & 19,2 \\
\hline $20: 0$ & 0,2 & 0,2 & 0,2 \\
\hline$\Sigma$ saturés & 34,8 & 18,4 & 47,6 \\
\hline $16: 1 \mathrm{n}-7$ & 3,7 & 10,3 & 1,7 \\
\hline $18: 1 \mathrm{n}-7$ & 2,6 & 4,5 & 2,1 \\
\hline $20: 1 n-7$ & 0,3 & 0,5 & 0,1 \\
\hline$\Sigma \mathrm{n}-7$ & 6,6 & 15,3 & 3,9 \\
\hline $18: 1 n-9$ & 18,1 & 15,1 & 31,2 \\
\hline $20: 1 n-9$ & 3,1 & 10,6 & 1,0 \\
\hline $22: 1 n-9$ & 0,4 & 0,6 & $\mathrm{t}$ \\
\hline$\Sigma n-9$ & 21,6 & 26,3 & 32,2 \\
\hline $18: 2 \mathrm{n}-6$ & 18,8 & 6,6 & 11,8 \\
\hline $18: 3 n-6$ & 0,1 & 0,1 & 0,1 \\
\hline $20: 2 n-6$ & 0,3 & 0,3 & 0,4 \\
\hline $20: 4 n-6$ & 0,5 & 0,5 & 0,2 \\
\hline $22: 5 n-6$ & 0,1 & 0,1 & 0,1 \\
\hline$\Sigma \mathrm{n}-6$ & 19,8 & 7,6 & 12,6 \\
\hline $18: 3 n-3$ & 2,6 & 1,3 & 1,1 \\
\hline $18: 4 n-3$ & 0,8 & 1,8 & $t$ \\
\hline $20: 4 n-3$ & 0,3 & 0,8 & 0,5 \\
\hline $20: 5 n-3$ & 4,7 & 9,4 & 0,2 \\
\hline $22: 5 n-3$ & 0,7 & 1,0 & $t$ \\
\hline $22: 6 n-3$ & 4,6 & 10,7 & 1,0 \\
\hline$\Sigma n-3$ & 13,7 & 25,0 & 2,8 \\
\hline
\end{tabular}

$\mathrm{C}=$ aliment commercial (Trouvit) contenant $8 \%$ de lipides ; $\mathrm{M}$ et $\mathrm{S}=$ aliments expérimentaux contenant respectivement $10 \%$ d'huile de foie de morue et de saindoux.

Analyse des échantillons. - Les muscles et les foies sont lyophilisés et leurs lipides totaux (LT) ainsi que ceux du tissu adipeux sont extraits selon la procédure décrite par Folch et al. (1957). La séparation des classes de lipides est effectuée sur colonne $(25 \times 2,5 \mathrm{~cm})$ remplie d'acide silicique et hyflosupercel dans les proportions $2: 1(\mathrm{P} / \mathrm{P})$. Les lipides neutres (LN) et les lipides polaires (LP) des échantillons déposés $(200 \mathrm{mg}$ de $\mathrm{LT}$ ) sont élués successivement par $500 \mathrm{ml}$ de chloroforme, puis par le même volume de méthanol. Les quantités des LT, LN et LP obtenues sont rapportées au poids sec, puis au poids frais du tissu. Les esters méthyliques des acides gras, préparés selon la procédure décrite antérieurement (Léger et al., 1981) sont analysés par chromatographie en phase gazeuse sur colonne capillaire en verre $(0,35 \mathrm{~mm}$ di $\times 50 \mathrm{~m})$ tapissée d'une phase stationnaire : la FFAP (Supelco Inc., Bellefonte, PA). Les températures de la colonne et de l'injecteur sont de $190^{\circ} \mathrm{C}$ et de $230{ }^{\circ} \mathrm{C}$ respectivement. 
TABLEAU 2

Composition des aliments expérimentaux.

\begin{tabular}{lc}
\hline \multicolumn{1}{c}{ Composants } & $\%$ \\
\hline Caséine & 15 \\
Farine de sang $_{\text {CPSP(a) }^{(\mathrm{a})}}$ & 5 \\
Blé $^{\text {Tourteau de soja }}$ & 10 \\
Lipides $^{(\mathrm{b})}$ & 30 \\
Complément vitaminique (c) $^{(\mathrm{c})}$ & 20 \\
Complément minéral $^{(\mathrm{d})}$ & 10 \\
Cellulose $^{\text {Lignosulfite }}$ & 2 \\
& 2 \\
\hline
\end{tabular}

(a) Concentré de protéines solubles de poisson contenant $83 \%$ de protéines par rapport au poids frais (solubilité de la protéine dans l'eau : 75 à $80 \%$ ). ${ }^{\text {(b) }}$ Huile de foie de morue ou saindoux. (c) (d) Les compositions du complément vitaminique et minéral sont rapportées antérieurement (Léger et al., 1981).

Traitement statistique. - Pour tous les paramètres étudiés, les calculs sont faits sur 6 échantillons par groupe. Les comparaisons de moyennes ont été effectuées par le test t de Student (Snedecor et Cochran, 1957).

\section{Résultats et discussion.}

Effets sur la matière sèche. - Les résultats du tableau 3 ne mettent pas en évidence d'effet des régimes alimentaires expérimentaux sur les teneurs en matière sèche du muscle et du foie. Toutefois, comparée à celle du régime préexpérimental $(\mathrm{C})$, la teneur en matière sèche diminue avec le régime carencé, alors qu'elle n'est pas modifiée avec l'aliment enrichi. Ces résultats semblent confirmer les travaux antérieurs indiquant que le régime déficient en AG n-3 ne modifie pas la teneur en matière sèche hépatique, mais entraîne une légère diminution de celle du tissu musculaire (Castell et al., $1972 \mathrm{c}$, Watanabe et al., 1974 a, b, c ; Takeuchi et Watanabe, 1976, 1982).

Effets sur les lipides tissulaires. - Les données du tableau 3 montrent que la teneur en LT du muscle est significativement plus faible chez les truites recevant le régime $S$ que celles soumises au régime $M$ et que cette baisse porte uniquement sur les LN, tandis que le taux des LP reste stable $(0,8 \%$ environ) quel que soit le régime. Cette observation concorde avec celle rapportée antérieurement chez la truite (Watanabe et al., 1974 a, b; Watanabe et Takeuchi, 1976), la daurade Chrysophrys major (Yone et Fujii, 1975) et l'anguille japonaise (Takeuchi et al., 1980). L'ensemble des résultats indiquent donc que la carence en AGE entraîne une diminution du contenu en LT consécutif à un abaissement des LN. Fukazawa et al. (1971) mettent en cause la diminution d'une forme de transport importante des triglycérides hépatiques, les VLDL, dans l'abaissement des lipides musculaires 


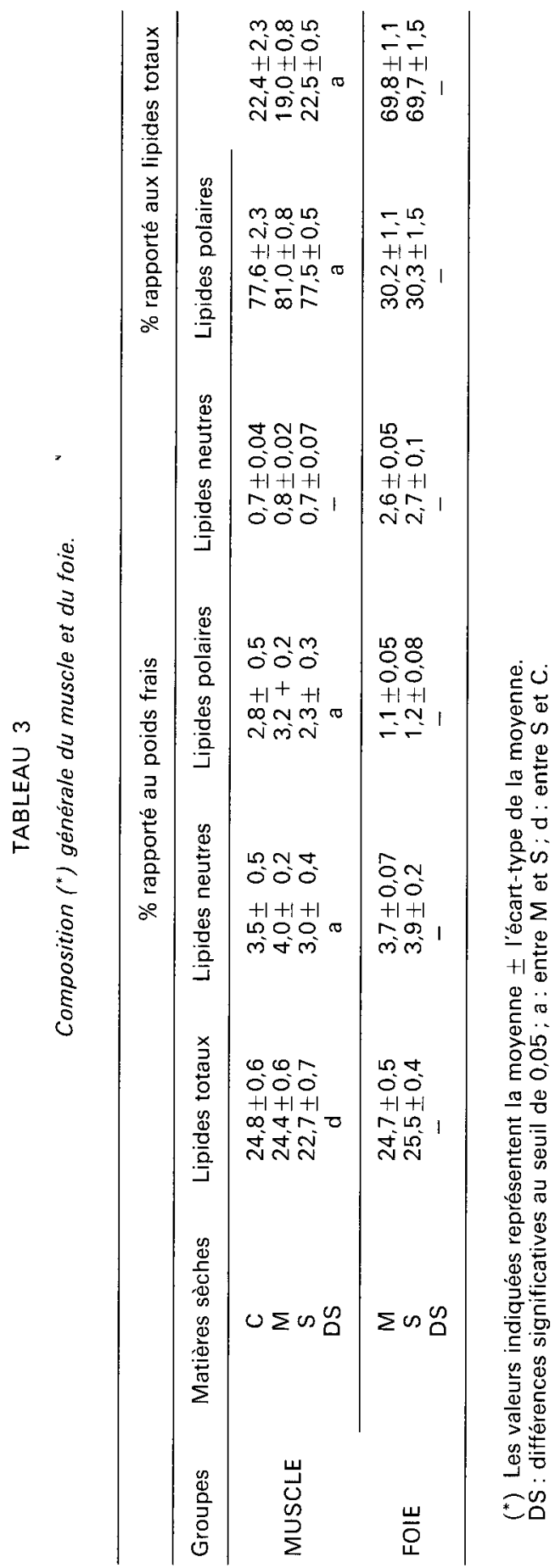


chez le rat carencé en AGE n-6. Cependant, chez la truite, Léger et al. (1981) et Frémont et al. (1984) ne montrent aucune relation entre le type de régime (carencé ou non) et le taux des différentes formes conventionnelles de transport des lipides (hors vitellogénine). L'analyse de la composition en acides gras des lipoprotéines laisse supposer que la diminution des lipides du muscle pourrait être due à une faible activité de la lipoprotéine-lipase vis-à-vis des triglycérides moins insaturés des lipoprotéines d'animaux carencés (Léger et al., 1981). C'est d'ailleurs ce que confirment Coiffier et al. (1987) chez des rats recevant un aliment supplémenté en acides gras saturés.

Chez les alevins, de nombreux travaux ont montré que le régime dépourvu d'AGE augmente le taux des LT hépatiques à la suite d'une élévation du taux de LN (Castell et al., 1972 a ; Watanabe et al., 1974 a ; Watanabe et Takeuchi, 1976). Par contre, chez la truite adulte, les données du tableau 3 confirment les travaux de Watanabe et al. (1984) et de Leray et al. (1985) et montrent que les taux des $L T, L N$ et LP hépatiques restent stables quel que soit le régime considéré. Cette absence d'effet du régime déficient sur les adultes ne semble pas due à la durée de l'expérience (Watanabe et Takeuchi, 1976) ou au stade physiologique des poissons (Nassour et Léger, données non publiées). Les travaux de Castell et al. (1972 c) et de Watanabe et al. (1974 c) laissent supposer que la qualité des lipides peut être en cause. Ces auteurs observent que chez les animaux carencés en AG n-3, l'accumulation des lipides dans le foie est plus importante chez les truites recevant $5 \%$ des lipides uniquement sous forme d'oléate ou de laurate que chez celles qui reçoivent un régime contenant $1 \%$ de $18: 2$ n- 6 avec $4 \%$ d'oléate ou de laurate. Dans notre expérience, comme dans celle de Watanabe et al. (1984) et de Leray et al. (1985), le taux élevé d'AG n-6 (> $12 \%$ ) présents dans le régime carencé pourrait donc avoir empêché l'accumulation des lipides dans le foie.

Effets sur les acides gras saturés. - En dépit d'une grande différence de composition de l'aliment, le taux des AG saturés des LP aussi bien que des LN varie peu d'un lot à l'autre (tabl. 4,5 et 6 ). Cette observation confirme les travaux antérieurs révélant une relative insensibilité au régime du taux des AG saturés tissulaires (Lee et al., 1967; Watanabe et Takeuchi, 1976; Yu et al., 1977; Mugritchian et al., 1981). Ces acides gras subissent une régulation qui explique probablement leur relative constance lorsque l'apport alimentaire est modifié. Toutefois, nos résultats laissent supposer que c'est au niveau des LP que cette régulation est la plus efficace.

Effets sur les $A G n-7$ et $A G n-9$. - Quel que soit le régime, les $A G n-7$ et $A G$ $\mathrm{n}-9$ sont incorporés préférentiellement dans les $L N$. Dans le muscle, le foie et le tissu adipeux, les taux des AG n-9 les plus élevés sont observés chez les animaux recevant le régime $S$, et pour les deux premiers tissus, dans les $L N$ aussi bien que dans les LP (tabl. 4, 5 et 6). Inversement, les taux des AG n-7 les plus élevés sont obtenus avec les animaux soumis au régime $M$. Ces modifications semblent résulter de l'influence directe des taux des $A G n-9$ et $A G n-7$ présents dans l'aliment. En toute rigueur ces modifications pourraient être également dues à une augmentation des synthèses de novo. II ne semble pas que cela soit le cas. II est 
TABLEAU 4

Composition $\left({ }^{*}\right)$ en acides gras du muscle.

\begin{tabular}{|c|c|c|c|c|c|c|c|c|}
\hline \multirow[t]{2}{*}{ Acides gras } & \multicolumn{4}{|c|}{ Lipides neutres } & \multicolumn{4}{|c|}{ Lipides polaires } \\
\hline & C & $M$ & $\mathrm{~s}$ & DS & C & $M$ & S & DS \\
\hline $\begin{array}{c}16: 0 \\
18: 0 \\
\Sigma \text { saturés }\end{array}$ & $\begin{array}{r}20,1 \pm 0,7 \\
5,3 \pm 0,2 \\
29,0 \pm 0,9\end{array}$ & $\begin{array}{r}17,1 \pm 0,4 \\
4,5 \pm 0,1 \\
24,9 \pm 0,5\end{array}$ & $\begin{array}{r}17,3 \pm 0,8 \\
5,4 \pm 0,1 \\
25,7 \pm 1,0\end{array}$ & $\begin{array}{l}b-d \\
a-b \\
b-d\end{array}$ & $\begin{array}{r}24,6 \pm 0,3 \\
5,1 \pm 0,1 \\
30,8 \pm 0,3\end{array}$ & $\begin{array}{r}21,2 \pm 0,1 \\
5,0 \pm 0,2 \\
27,4 \pm 0,3\end{array}$ & $\begin{array}{r}21,6 \pm 0,3 \\
5,1 \pm 0,3 \\
27,7 \pm 0,2\end{array}$ & $\begin{array}{l}b-d \\
- \\
b-d\end{array}$ \\
\hline $\begin{array}{c}16: 1 \mathrm{n}-7 \\
18: 1 \mathrm{n}-7 \\
\Sigma \mathrm{n}-7\end{array}$ & $\begin{array}{l}5,9 \pm 0,1 \\
2,1 \pm 0,07 \\
8,3 \pm 0,2\end{array}$ & $\begin{array}{r}7,8 \pm 0,4 \\
2,3 \pm 0,1 \\
10,3 \pm 0,3\end{array}$ & & $\begin{array}{r}a-b-d \\
a-b \\
a-b-d\end{array}$ & & $\begin{array}{l}1,4 \pm 0,04 \\
1,6 \pm 0,09 \\
2,9 \pm 0,1\end{array}$ & $\begin{array}{l}0,9 \pm 0,02 \\
1,1 \pm 0,04 \\
2,0 \pm 0,04\end{array}$ & $\begin{array}{r}a-b \\
a-b-d \\
a-b\end{array}$ \\
\hline $\begin{array}{c}18: 1 n-9 \\
20: 1 \mathrm{n}-9 \\
\Sigma n-9\end{array}$ & $\begin{array}{r}20,4 \pm 0,3 \\
3,1 \pm 0,1 \\
23,9 \pm 0,4\end{array}$ & $\begin{array}{r}19,7 \pm 1,0 \\
4,0 \pm 0,5 \\
24,2 \pm 0,5\end{array}$ & $\begin{array}{r}24,5 \pm 0,6 \\
3,7 \pm 0,2 \\
28,7 \pm 0,7\end{array}$ & $\begin{array}{r}a-d \\
d \\
a-d\end{array}$ & $\begin{array}{l}5,8 \pm 0,3 \\
0,4 \pm 0,02 \\
6,2 \pm 0,3\end{array}$ & & $\begin{array}{l}7,0 \pm 0,3 \\
0,5 \pm 0,06 \\
7,5 \pm 0,3\end{array}$ & $\begin{array}{c}a-b \\
- \\
a-d\end{array}$ \\
\hline $\begin{array}{c}18: 2 n-6 \\
20: 2 \text { n-6 } \\
20: 3 n-6 \\
20: 4 \text { n-6 } \\
22: 5 n-6 \\
\Sigma n-6 \\
\Sigma n-6 \Delta 6\end{array}$ & $\begin{array}{c}16,9 \pm 0,4 \\
1,2 \pm 0,08 \\
0,9 \pm 0,06 \\
0,9 \pm 0,07 \\
0,4 \pm 0,03 \\
20,8 \pm 0,4 \\
2,7 \pm 0,1\end{array}$ & $\begin{array}{c}16,4 \pm 0,5 \\
1,1 \pm 0,04 \\
0,8 \pm 0,04 \\
0,9 \pm 0,05 \\
0,3 \pm 0,02 \\
19,9 \pm 0,5 \\
2,4 \pm 0,1\end{array}$ & $\begin{array}{c}18,6 \pm 0,7 \\
1,4 \pm 0,05 \\
1,0 \pm 0,04 \\
1,2 \pm 0,1 \\
0,6 \pm 0,04 \\
23,6 \pm 0,8 \\
3,6 \pm 0,1\end{array}$ & $\begin{array}{r}a-d \\
a \\
a-d \\
a-d \\
a-b-d \\
a-d \\
a-d\end{array}$ & $\begin{array}{c}5,6 \pm 0,3 \\
0,6 \pm 0,07 \\
1,0 \pm 0,04 \\
3,2 \pm 0,1 \\
0,9 \pm 0,07 \\
11,5 \pm 0,3 \\
5,3 \pm 0,2\end{array}$ & $\begin{array}{c}6,6 \pm 0,7 \\
0,6 \pm 0,03 \\
1,0 \pm 0,06 \\
3,2 \pm 0,07 \\
0,6 \pm 0,03 \\
12,1 \pm 0,8 \\
5,0 \pm 0,2\end{array}$ & $\begin{array}{c}8,4 \pm 0,3 \\
0,7 \pm 0,04 \\
1,6 \pm 0,1 \\
4,8 \pm 0,2 \\
1,3 \pm 0,09 \\
17,3 \pm 0,2 \\
8,2 \pm 0,3\end{array}$ & $\begin{array}{r}a-d \\
a \\
a-d \\
a-d \\
a-b-d \\
a-d \\
a-d\end{array}$ \\
\hline $\begin{array}{c}18: 3 n-3 \\
20: 4 n-3 \\
20: 5 n-3 \\
22: 5 n-3 \\
22: 6 n-3 \\
\Sigma n-3\end{array}$ & $\begin{array}{c}1,3 \pm 0,03 \\
0,4 \pm 0,02 \\
2,3 \pm 0,2 \\
1,1 \pm 0,07 \\
9,4 \pm 0,4 \\
14,9 \pm 0,5\end{array}$ & $\begin{array}{c}1,4 \pm 0,03 \\
0,5 \pm 0,04 \\
2,9 \pm 0,4 \\
1,6 \pm 0,09 \\
10,3 \pm 0,6 \\
17,3 \pm 1,0\end{array}$ & $\begin{array}{c}1,2 \pm 0,05 \\
0,3 \pm 0,02 \\
1,6 \pm 0,2 \\
1,2 \pm 0,1 \\
7,7 \pm 0,3 \\
12,3 \pm 0,6\end{array}$ & $\begin{array}{l}a-d \\
a-b \\
a-d \\
a-b \\
a-d \\
a-d\end{array}$ & $\begin{array}{c}0,6 \pm 0,06 \\
0,3 \pm 0,03 \\
5,9 \pm 0,2 \\
1,4 \pm 0,07 \\
40,7 \pm 0,2 \\
48,9 \pm 0,3\end{array}$ & $\begin{array}{c}0,7 \pm 0,03 \\
0,4 \pm 0,02 \\
8,5 \pm 0,2 \\
2,2 \pm 0,06 \\
38,6 \pm 0,7 \\
50,6 \pm 0,6\end{array}$ & $\begin{array}{c}0,7 \pm 0,03 \\
0,3 \pm 0,02 \\
5,0 \pm 0,3 \\
1,6 \pm 0,09 \\
36,8 \pm 0,8 \\
44,5 \pm 0,7\end{array}$ & $\begin{array}{r}- \\
a-b \\
a-b-d \\
a-b \\
b-d \\
a-b-d\end{array}$ \\
\hline
\end{tabular}

$\left({ }^{*}\right)$ Les valeurs indiquées représentent la moyenne \pm l'écart-type de la moyenne. et $\mathrm{C}$.

DS : différences significatives au seuil de 0,05 ; a : entre $M$ et $S$; b : entre $M$ et $C$; $d$ : entre $S$

$\Sigma(n-6)_{\Delta 6}=18: 3(n-6)+20: 3(n-6)+20: 4(n-6)+22: 5(n-6)$, acides gras polyinsaturés $n-6$ issus de la désaturation en $\Delta 6$.

en revanche probable que la présence de quantités importantes d'AG n-6 dans le régime même carencé en $A G n-3$ soit suffisante pour bloquer la synthèse de novo des AG n-7 et AG n-9 (Jeffcoat et James, 1984). L'absence du produit ultime de la bioconversion du $18: 1 n-9$, le $20: 3 n-9$, est de toute façon la preuve que les $\Delta 6$ - et $\Delta 5$-désaturases sont bloquées dans le type de carence réalisé dans cette expérience.

Effets sur les acides gras polyinsaturés. - Comparé au lot préexpérimental (lot $C$ ), le taux des $A G n-3$ des $L N$ du muscle augmente avec le régime $M$ et diminue avec le régime $S$ (tabl. 4). Cependant, au niveau du tissu adipeux, à l'exception des acides gras minoritaires (18:3n-3 et $20: 4 n-3)$, les taux des AG $\mathrm{n}-3$ dans les lots $M$ et $S$ restent comparables à ceux du lot $C$ (tabl. 6 ). Ces résultats indiquent qu'au cours des 2 derniers mois de la vitellogenèse, les régimes alimentaires n'exercent qu'un faible effet sur le niveau des $A G n-3$ du tissu adipeux. Cette observation a d'autant plus de valeur que, à l'inverse, les mêmes régimes affectent profondément la composition en $A G$ n-3 de ce tissu en période 
TABLEAU 5

Composition (*) en acides gras du foie.

\begin{tabular}{|c|c|c|c|c|c|c|}
\hline \multirow{2}{*}{ Acides gras } & \multicolumn{3}{|c|}{ Lipides neutres } & \multicolumn{3}{|c|}{ Lipides polaires } \\
\hline & $M$ & S & DS & $M$ & s & DS \\
\hline $16: 0$ & $16,9 \pm 0,5$ & $18,3 \pm 0,4$ & $\mathrm{a}$ & $16,5 \pm 0,7$ & $16,8 \pm 0,7$ & - \\
\hline $18: 0$ & $5,2 \pm 0,3$ & $6,6 \pm 0,3$ & $a$ & $9,3 \pm 0,5$ & $10,4 \pm 0,5$ & - \\
\hline$\Sigma$ saturés & $24,0 \pm 0,7$ & $27,1 \pm 0,3$ & $\mathrm{a}$ & $27,0 \pm 0,5$ & $28,8 \pm 0,7$ & - \\
\hline $16: 1 n-7$ & $4,7 \pm 0,2$ & $3,8 \pm 0,2$ & $a$ & $1,9 \pm 0,2$ & $2,0 \pm 0,06$ & - \\
\hline $18: 1 n-7$ & $4,1 \pm 0,3$ & $3,2 \pm 0,2$ & $a$ & $4,1 \pm 0,2$ & $3,3 \pm 0,3$ & a \\
\hline$\Sigma n-7$ & $8,8 \pm 0,4$ & $7,0 \pm 0,2$ & $a$ & $6,0 \pm 0,4$ & $5,3 \pm 0,3$ & - \\
\hline $18: 1 n-9$ & $13,2 \pm 0,4$ & $17,6 \pm 1,1$ & $\mathrm{a}$ & $9,4 \pm 0,3$ & $11,0 \pm 0,4$ & a \\
\hline $20: 1 n-9$ & $1,3 \pm 0,2$ & $1,1 \pm 0,2$ & - & $1,4 \pm 0,2$ & $1,1 \pm 0,05$ & - \\
\hline$\Sigma n-9$ & $14,5 \pm 0,5$ & $18,7 \pm 1,1$ & $\mathrm{a}$ & $10,7 \pm 0,3$ & $12,1 \pm 0,4$ & a \\
\hline $18: 2 n-6$ & $9,2 \pm 0,5$ & $8,7 \pm 0,8$ & - & $5,6 \pm 0,4$ & $6,9 \pm 0,5$ & - \\
\hline $20: 2$ n-6 & $0,9 \pm 0,1$ & $1,3 \pm 0,1$ & $a$ & $1,3+0,1$ & $1,4+0,2$ & - \\
\hline $20: 3 n-6$ & $0,9 \pm 0,04$ & $1,6 \pm 0,1$ & $\mathrm{a}$ & $0,9 \pm 0,1$ & $2,2 \pm 0,3$ & $a$ \\
\hline $20: 4 n-6$ & $5,6 \pm 0,3$ & $8,4 \pm 0,8$ & a & $6,7 \pm 0,6$ & $11,2 \pm 1,0$ & a \\
\hline $22: 5 n-6$ & $0,1 \pm 0,02$ & $1,0 \pm 0,2$ & $a$ & $0,2 \pm 0,02$ & $0,8 \pm 0,2$ & a \\
\hline$\sum n-6$ & $16,9 \pm 0,6$ & $21,7 \pm 0,6$ & a & $14,9 \pm 0,7$ & $23,2 \pm 1,1$ & a \\
\hline$\sum n-6_{\Lambda 6}$ & $6,8 \pm 0,3$ & $11,7 \pm 1,0$ & $\mathrm{a}$ & $7,9 \pm 0,6$ & $14,8 \pm 1,4$ & a \\
\hline $18: 3 n-3$ & $1,0 \pm 0,04$ & $0,5 \pm 0,05$ & a & $0,4 \pm 0,06$ & $0,2 \pm 0,01$ & a \\
\hline $20: 4 n-3$ & $0,4 \pm 0,05$ & $0,3 \pm 0,05$ & $a$ & $0,2 \pm 0,02$ & $0,1 \pm 0,02$ & - \\
\hline $20: 5 n-6$ & $8,9 \pm 0,8$ & $3,4 \pm 0,3$ & a & $8,2 \pm 0,8$ & $3,5+0,3$ & a \\
\hline $22: 5 n-3$ & $2,2+0,08$ & $1,1+0,07$ & a & $2,0+0,2$ & $0,9+0,07$ & a \\
\hline $22: 6 \mathrm{n}-3$ & $218+0,9$ & $18,8+1,1$ & - & $30.1+1.0$ & $25,0+0,6$ & a \\
\hline$\sum n-3$ & $346+14$ & $243+14$ & $\mathrm{a}$ & $409+07$ & $297+0.6$ & a \\
\hline
\end{tabular}

(*) Les valeurs indiquées représentent la moyenne \pm l'écart-type de la moyenne. $S$ et $C$

DS : différences significatives au seuil de $0,05: a$ : entre $M$ et $S ; b$ : entre $M$ et $C ; d$ : entre

$\Sigma(n-6)_{\Delta 6}=18: 3(n-6)+20: 3(n-6)+20: 4(n-6)+22: 5(n-6)$, acides gras polyinsaturés $n-6$ issus de la désaturation en $\Delta 6$.

de repos sexuel (Léger et al., 1977). Ces résultats semblent en faveur d'une modification de la fonction du tissu adipeux au cours du cycle sexuel. En période de repos sexuel, le tissu adipeux pourrait être le siège d'un dépôt intensif des lipides alimentaires, tandis qu'au cours des 2 derniers mois de la vitellogenèse ce dépôt est peu important. La mobilisation des acides gras à partir de ce tissu l'emporte sur le stockage (Ando et al., 1985). La modification de la fonction du tissu adipeux pourrait résulter du blocage de la lipoprotéine-lipase par les taux élevés d'oestrogènes plasmatiques (Hamosh et Hamosh, 1975 ; Van Bohemen et Lambert, 1981). Ces résultats suggèrent également que la carence alimentaire en AG n-3 ne semble pas être compensée par une mobilisation sélective des AGE du tissu adipeux, ce qui confirme les données obtenues chez les poissons subissant un jeûne prolongé (Dave et al., 1976 ; Murata et Higashi, 1980; Léger, 1981 ; Ando et al., 1985).

Quel que soit le lot considéré, les acides gras n-6 issus de la désaturation en $\Delta 6\left(A G n-6_{\Delta 6}\right)$ présentent dans les tissus musculaire, hépatique et adipeux des taux plus élevés que dans les régimes correspondants (tabl. 2, 4, 5, 6). Ceci 
TABLEAU 6

Composition (*) en acides gras du tissu adipeux.

\begin{tabular}{|c|c|c|c|c|}
\hline \multirow{2}{*}{ Acides gras } & \multicolumn{4}{|c|}{ Tissu adipeux } \\
\hline & c & $M$ & $\mathrm{~s}$ & DS \\
\hline $\begin{array}{c}16: 0 \\
18: 0 \\
\Sigma \text { saturés }\end{array}$ & $\begin{array}{r}16,3 \pm 0,9 \\
5,2 \pm 0,1 \\
25,6 \pm 0,9\end{array}$ & $\begin{array}{r}11,8 \pm 0,4 \\
4,0 \pm 0,3 \\
20,0 \pm 0,5\end{array}$ & $\begin{array}{c}13,3 \pm 0,8 \\
5,5 \pm 0,05 \\
22,3 \pm 0,7\end{array}$ & $\begin{array}{l}b-d \\
a-b-d \\
a-b-d\end{array}$ \\
\hline $\begin{array}{c}16: 1 n-7 \\
18: 1 n-7 \\
\Sigma n-7\end{array}$ & $\begin{array}{c}6,8 \pm 0,3 \\
3,2 \pm 0,06 \\
10,3 \pm 0,3\end{array}$ & $\begin{array}{c}7,5 \pm 0,6 \\
3,6 \pm 0,08 \\
11,5 \pm 0,6\end{array}$ & $\begin{array}{l}4,0 \pm 0,2 \\
3,0 \pm 0,07 \\
7,3 \pm 0,3\end{array}$ & $\begin{array}{r}a-d \\
a-b-d \\
a-d\end{array}$ \\
\hline $\begin{array}{c}18: 1 n-9 \\
20: 1 n-9 \\
\Sigma n-9\end{array}$ & $\begin{array}{r}26,0 \pm 0,5 \\
3,6 \pm 0,3 \\
30,1 \pm 0,7\end{array}$ & $\begin{array}{r}26,6 \pm 0,7 \\
5,3 \pm 0,4 \\
32,5 \pm 0,7\end{array}$ & $\begin{array}{r}29,5 \pm 1,3 \\
4,8 \pm 0,5 \\
35,0 \pm 1,6\end{array}$ & $\begin{array}{r}d \\
b \\
b-d\end{array}$ \\
\hline $\begin{array}{c}18: 2 n-6 \\
20: 2 n-6 \\
20: 3 n-6 \\
20: 4 n-6 \\
22: 5 n-6 \\
\Sigma n-6 \\
\sum n-66_{\Delta 6}\end{array}$ & $\begin{array}{c}17,8 \pm 0,8 \\
1,4 \pm 0,1 \\
0,7 \pm 0,04 \\
0,6 \pm 0,03 \\
0,2 \pm 0,02 \\
21,1 \pm 0,8 \\
1,9 \pm 0,06\end{array}$ & $\begin{array}{c}17,5 \pm 0,6 \\
1,2 \pm 0,05 \\
0,7 \pm 0,02 \\
0,6 \pm 0,03 \\
0,2 \pm 0,02 \\
20,5 \pm 0,7 \\
1,8 \pm 0,07\end{array}$ & $\begin{array}{c}17,5 \pm 0,8 \\
2,2 \pm 0,5 \\
1,2 \pm 0,09 \\
1,8 \pm 0,6 \\
0,3 \pm 0,06 \\
23,6 \pm 1,0 \\
3,8 \pm 0,6\end{array}$ & $\begin{array}{r}- \\
- \\
\text { a-d } \\
- \\
- \\
\text { a } \\
\text { a-d }\end{array}$ \\
\hline $\begin{array}{l}18: 3 n-3 \\
20: 4 n-3 \\
20: 5 n-3 \\
22: 5 n-3 \\
22: 6 n-3\end{array}$ & $\begin{array}{l}1,3 \pm 0,02 \\
0,3 \pm 0,02 \\
1,3 \pm 0,09 \\
1,1 \pm 0,07 \\
5,6 \pm 0,4\end{array}$ & $\begin{array}{l}1,2 \pm 0,08 \\
0,5 \pm 0,04 \\
1,8 \pm 0,3 \\
1,7 \pm 0,1 \\
5,6 \pm 0,5\end{array}$ & $\begin{array}{l}0,7 \pm 0,05 \\
0,2 \pm 0,02 \\
1,0 \pm 0,3 \\
1,5 \pm 0,1 \\
5,0 \pm 1,2\end{array}$ & $\begin{array}{r}a-d \\
a-b-d \\
- \\
b-d \\
-\end{array}$ \\
\hline$\Sigma n-3$ & $10,2 \pm 0,4$ & $11,5 \pm 0,8$ & $8,7 \pm 1,6$ & - \\
\hline
\end{tabular}

${ }^{*}{ }^{*}$ Les valeurs indiquées représentent la moyenne \pm l'écart-type de la moyenne. $S$ et $C$.

DS : différences significatives au seuil de 0,$05 ; a$ : entre $M$ et $S ; b$ : entre $M$ et $C ; d$ : entre

$\Sigma(n-6)_{\wedge 6}=18: 3(n-6)+20: 3(n-6)+20: 4(n-6)+22: 5(n-6)$, acides gras polyinsaturés $n-6$ issus de la désaturation en $\Delta 6$.

témoigne de la capacité de la truite à bioconvertir le 18:2 n-6 en AGLPI n-6 mentionnés antérieurement (Castell et al., 1972 a; Yu et Sinnhuber, 1972). Toutefois, nos résultats montrent que cette élévation du taux des $A G n-6_{\Delta 6}$ est plus importante dans le lot $S$ que dans les lots $M$ et $C$ et tout particulièrement dans la fraction LP (tabl. 4, 5 et 6 ). II semble donc que la truite compense la carence en $A G$ n-3 par une bioconversion accrue des $A G n-6$ qui seront ensuite préférentiellement incorporés dans les LP. En effet, au niveau des LP hépatiques et musculaires, on observe un phénomène de compensation entre $A G n-3$ et $A G$ $n-6$ permettant de maintenir les acides gras polyinsaturés totaux $(n-3+n-6)$ à un taux constant. Au niveau des LN, l'abaissement des taux des $A G n-3$ dû à la carence est compensé par l'augmentation des AG n-6 et AG n-9.

Castledine et Buckley (1982) montrent que les phospholipides totaux des truites carencées en $A G n-3$ pendant 10 semaines présentent une baisse importante du taux des $A G \mathrm{n}-3$ allant de 50 à $76 \%$, selon la classe de phospholipides. Dans notre expérience, la baisse du taux des AG n-3 est 
beaucoup plus faible, sur une durée comparable. De plus, une durée triple de la carence (Nassour et Léger, données non publiées) ne permet pas d'atteindre une chute analogue à celle qui est rapportée par ces auteurs. Cette différence majeure entre les deux études pourrait être liée au stade de développement. Castledine et Buckley (1982) ont analysé de jeunes animaux (40 à $100 \mathrm{~g}$ ). Ceux-ci, à l'opposé des adultes (notre expérience) ont un taux de croissance plus élevé et disposent par ailleurs de réserves lipidiques plus faibles. L'un ou l'autre de ces facteurs, ou leur conjonction, sont de nature à expliquer l'effet marqué de la carence alimentaire en $A G n-3$ sur le niveau d'AG n-3 dans les phospholipides chez les jeunes animaux et l'effet notablement plus faible de cette carence sur des animaux adultes.

Reçu en mai 1988

Accepté en août 1988

\section{References}

ANDO S., HATANO M., ZAMA K., 1985. A consumption of muscle lipid during spawning migration of chum salmon Oncorhynchus keta. Bull. jpn. Soc. sci. Fish., 51, 1817-1824.

CASTELL J. D., 1979. Review of lipid requirements of finfish. In Proc. World Symp. on Finfish nutrition and fishfeed technology. Hamburg, 20-23 June 1978, Berlin, vol. 1, p. 59-84.

CASTELL J. D., LEE D. J., SINNHUBER R. O, 1972 a. Essential fatty acids in the diet of rainbow trout (Salmo gairdneri) : Lipid metabolism and fatty acid composition. J. Nutr., 102, 93-100.

CASTELL J. D., SINNHUBER R. O., LEE D. J., WALES J. H., 1972 b. Essential fatty acids in the diet of rainbow trout (Salmo gairdnerii): Physiological symptoms of essential fatty acid deficiency. J. Nutr., 102, 87-92.

CASTELL J. D., SINNHUBER R. O., WALES J. H., LEE D. J., 1972 c. Essential fatty acids in the diet of rainbow trout (Salmo gairdnerii): Growth, feed conversion and some gross deficiency symptoms. J. Nutr., 102, 77-85.

CASTLEDINE A. J., BUCKLEY J. Y., 1982. Incorporation and turnover of essential fatty acids in phospholipids and neutral lipids of rainbow trout. Comp. Biochem. Physiol., 71 B, 119-126.

COIFFIER E., PARIS R., LECERF J., 1987. Effects of dietary saturated and polyunsaturated fat on lipoprotein lipase and hepatic triglyceride lipase activity. Comp. Biochem. Physiol., 88 B, 187-192

DAVE G., JOHANSSON-SJOBECK M. L., LARSSON A., LEWANDER K., LIDMAN U., 1976 . Metabolic and hematological effects of starvation in the European eel, Anguilla anguilla L. -III. Fatty acid composition. Comp. Biochem. Physiol., 53 B, 509-515.

FOLCH J., LEES M., STANLEY G. H. S., 1957. A simple method for the isolation and purification of total lipids from animal tissues. J. biol. Chem., 226, 497-509.

FRÉMONT L., LEGER C., PETRIDOU B., GOZZELINO M.-T., 1984. Effects of a $(n-3)$ polyunsaturated fatty acid-deficient diet on profiles of serum vitellogenin and lipoprotein in vitellogenic trout (Salmo gairdneri), Lipids, 19, 522-528.

FUKAZAWA T., PRIVETT O. S., TAKAHASHI Y., 1971. Effect of EFA deficiency on lipid transport from liver. Lipids, 6, 388-393.

HAMOSH M., HAMOSH P., 1975. The effect of estrogen on the lipoprotein lipase activity of rat adipose tissue. $J$. clin. Invest, 55, 1132-1135.

JEFFCOAT R., JAMES A. T., 1984. The regulation of desaturation and elongation of fatty acids in mammals. In S. NUMA. New comprehensive biochemistry, fatty acid metabolism and its regulation. Elsevier, Amsterdam, New York, Oxford, vol. 7, p. 85-112.

LEE D. J., ROEHM J. N., YU T. C., SINNHUBER R. O., 1967. Effect of $w 3$ fatty acids on the growth rate of rainbow trout Salmo gairdneri. J. Nutr., 92, 93-98. 
LÉGER C., 1980. Développements récents de la notion d'acides gras essentiels chez les poissons. Ann. Nutr. Alim. 34, 207-216.

LÉGER C., 1981. Effet du jeûne prolongé sur la composition en lipides et en acides gras de la truite arc-en-ciel Salmo gairdneri. Aquaculture, 25, 195-206.

LEGER C., BERGOT P., LUOUET P., FLANZY J., MEUROT J., 1977. Specific distribution of fatty acids in the triglycerides of rainbow trout adipose tissue. Influence of temperature. Lipids, 12, 538-543.

LÉGER C., FREMONT L., MARION D., NASSOUR I., DESFARGES M.-F., 1981. Essential fatty acids in trout serum, lipoproteins, vitellogenin and egg lipids. Lipids, 16, 593-600.

LÉGER C., GATESOUPE F. J., METAILLER R., LUOUET P., FREMONT L., 1979. Effect of dietary fatty acids differing by chain lengths and $w$ series on the growth and lipid composition of turbot Scophtalmus maximus L. Comp. Biochem. Physiol., 64 B, 345-350.

LERAY C., NONNOTTE G., ROUBAUD P., LÉGER C., 1985. Incidence of (n-3) essential fatty acid deficiency on trout reproductive processes. Reprod. Nutr. Dévelop., 25, 567-581.

MUGRIDTCHIAN D. S., HARDY R. W., IWAOKA W. T., 1981. Linseed oil and animal fat as alternative lipid sources in dry diets for Chinook salmon (Oncorhynchus tschawytscha). Aquaculture, 25, $161-172$.

MURATA H., HIGASHI T., 1980. Selective utilization of fatty acid as energy source in carp. Bull. jap. Soc. Sci. Fish, 46, 1333-1338.

OWEN J. M., ADRON J. W., MIDDLETON C., COWEY C. B., 1975. Elongation and desaturation of dietary fatty acids in turbot Scophthalmus maximus $\mathrm{L}$. and rainbow trout. Salmo gairdnerii Rich. Lipids, 10, 528-531.

SNEDECOR G. W., COCHRAN W. G., 1957. Méthodes statistiques. A.C.T.A., Paris, p. 100-128.

TAKEUCHI T., ARAI S., WATANABE T., SHIMMA Y., 1980. Requirement of eel Anguilla japonica for essential fatty acids. Bull. jpn. Soc. sci. Fish., 46, 345-353.

TAKEUCHI T., WATANABE T., 1976. Nutritive value of $w 3$ highly unsaturated fatty acids in pollock liver oil for rainbow trout. Bull. jpn. Soc. sci. Fish., 42, 907-919.

TAKEUCHI T., WATANABE T., 1977 a. Dietary levels of methyl laurate and essential fatty acid requirement of rainbow trout. Bull. jpn. Soc. sci. Fish., 43, 947-953.

TAKEUCHI T., WATANABE T., 1977 b. Effect of eicosapentaenoic acid and docosahexaenoic acid in pollock liver oil on growth and fatty acid composition of rainbow trout. Bull.jpn. Soc. Sci. Fish., 43, 947-953.

TAKEUCHI T., WATANABE T., 1982. Effects of various polyunsaturated fatty acids on growth and fatty acid compositions of rainbow trout Salmo gairdneri, coho salmon Onchorhynchus kisutch, and chum salmon Onchorhynchus keta. Bull. jpn. Soc. sci. Fish., 48, 1745-1752.

VAN BOHEMEN C. G., LAMBERT J. G. D., 1981. Estrogen synthesis in relation to estrone, estradiol and vitellogenin plasma levels during the reproductive cycle of the female rainbow trout, Salmo gairdneri. Gen. comp. Endocrinol., 45, 105-114.

WATANABE T., KOBAYASHI I., UTSIE O., OGINO C., 1974 a. Effect of dietary linolenate on fatty acid composition of lipids in rainbow trout. Bull. jpn. Soc. sci. Fish., 40, 387-392.

WATANABE T., OGINO C., KOSHIISHI Y., MATSUNAGA T., 1974 b. Requirement of rainbow trout for essential fatty acids. Bull. jpn. Soc. sci. Fish., 40, 493-499.

WATANABE T., TAKASHIMA F., OGINO C., $1974 \mathrm{c}$. Effect of dietary methyl linolenate on growth of rainbow trout. Bull. jph. Soc. sci. Fish., 40, 181-188.

WATANABE T., TAKEUCHI T., 1976. Evaluation of pollock liver oil as a supplement to diets for rainbow trout. Bull. jpn. Soc. sci. Fish., 42, 893-906.

WATANABE T., TAKEUCHI T., OGINO C., 1975. Effect of dietary methyl linoleate and linolenate on growth of carp. II. Bull. jpn. Soc. sci. Fish., 41, 263-269.

WATANABE T., TAKEUCHI T., SAITO M., NISHIMURA K., 1984. Effect of low protein-high calory or essential fatty acid deficient diet on reproduction of rainbow trout. Bull. jpn. Soc. sci. Fish., 50, 1207-1215.

YONE Y., FUJII M., 1975. Studies on nutrition of red sea bream. XII. Effect of w 3 fatty acid supplement in a corn oil diet on fatty acid composition of fish. Bull. jpn. Soc. sci. Fish., 41, $79-86$.

YU T. C., SINNHUBER R. O., 1972. Effect of dietary linolenic acid and docosahexaenoic acid on growth and fatty acid composition of rainbow trout (Salmo gairdneri). Lipids, 10, 63-66. 
YU T. C., SINNHUBER R. O., 1975. Effect of dietary linolenic and linoleic acids upon growth and lipid metabolism of rainbow trout (Salmo gairdneri). Lipids, 10, 63-66.

YU T. C., SINNHUBER R. O., HENDRICKS J. D., 1979. Reproduction and survival of rainbow trout (Salmo gairdneri) fed linolenic acid as the only source of essential fatty acids. Lipids, 14, $572-575$.

YU T. C., SINNHUBER R. O., PUTNAM G. B., 1977. Effect of dietary lipids on fatty acid composition of body lipid in rainbow trout (Sa/mo gairdneri). Lipids, 12, 495-499. 